\title{
Long-term spectroscopic monitoring of LBVs and LBV candidates
}

\author{
Alex Lobel ${ }^{1}$, Jose H. Groh ${ }^{2}$, Kelly Torres ${ }^{1}$ and Nadya Gorlova ${ }^{3}$ \\ ${ }^{1}$ Royal Observatory of Belgium \\ Ringlaan 3, B-1180, Brussels, Belgium \\ email: Alex.Lobel@oma.be; alobel@sdf.lonestar.org; Kelly.Torres@oma.be \\ ${ }^{2}$ Max Planck Institute for Radio Astronomy, \\ Auf dem Hügel 69, 53121 Bonn, Germany \\ email: jgroh@mpifr-bonn.mpg.de \\ ${ }^{3}$ Institute of Astronomy, Katholieke Universiteit Leuven, \\ Celestijnenlaan 200D BUS 2401, 3001 Leuven, Belgium \\ email: nadya@ster.kuleuven.be
}

\begin{abstract}
We present results of a long-term spectroscopic monitoring program (since mid 2009) of Luminous Blue Variables with the new HERMES echelle spectrograph on the $1.2 \mathrm{~m}$ Mercator telescope at La Palma (Spain). We investigate high-resolution $(R=80,000)$ optical spectra of two LBVs, P Cyg and HD 168607, the LBV candidates MWC 930 and HD 168625, and the LBV binary MWC 314. In P Cyg we observe flux changes in the violet wings of the Balmer $\mathrm{H} \alpha, \mathrm{H} \beta$, and He I lines between May and Sep 2009. The changes around 200 to $300 \mathrm{~km} \mathrm{~s}^{-1}$ are caused by variable opacity at the base of the supersonic wind from the blue supergiant.

We observe in MWC 314 broad double-peaked metal emission lines with invariable radial velocities over time. On the other hand, we measure in the photospheric S II $\lambda 5647$ absorption line, with lower excitation energy of $\sim 14 \mathrm{eV}$, an increase of the heliocentric radial velocity centroid from $37 \mathrm{~km} \mathrm{~s}^{-1}$ to $70 \mathrm{~km} \mathrm{~s}^{-1}$ between 5 and 10 Sep 2009 (and $43 \mathrm{~km} \mathrm{~s}^{-1}$ on $6 \mathrm{Apr}$ 2010). The increase of radial velocity of $\sim 33 \mathrm{~km} \mathrm{~s}^{-1}$ in only 5 days can confirm the binary nature of this LBV close to the Eddington luminosity limit.

A comparison with VLT-UVES and Keck-Hires spectra observed over the past 13 years reveals strong flux variability in the violet wing of the $\mathrm{H} \alpha$ emission line of $\mathrm{HD} 168625$ and in the absorption portion of the $\mathrm{H} \beta$ line of HD 168607. In HD 168625 we observe $\mathrm{H} \alpha$ wind absorption at velocities exceeding $200 \mathrm{~km} \mathrm{~s}^{-1}$ which develops between Apr and June 2010.
\end{abstract}

Keywords. stars: emission-line, stars: individual (P Cyg, MWC 314, MWC 930, HD 168607, HD 168625), stars: variables: other

\section{Introduction}

The HERMES instrument on the 1.2m Mercator telescope at La Palma (Raskin \& Van Winckel 2008) is a new high-efficiency fiber-fed bench-mounted cross-dispersed echelle spectrograph that observes the complete wavelength range from $420 \mathrm{~nm}$ to $900 \mathrm{~nm}$ in a single exposure with $R=80,000$. TAC reviewed HERMES observation programs of the contributing research institutions started mid 2009. We present first results of a longterm high-resolution spectroscopic monitoring program of 3 LBVs and 2 LBV candidates (up to $V=11^{\mathrm{m}} .0$ ). The HERMES monitoring program will provide invaluable new clues about the structure and dynamics of LBV atmospheres, the physics of their extended winds, and the strong line broadening mechanisms in these rare massive hot stars near the Eddington luminosity limit. The monitoring program will be crucial for documenting the enigmatic LBV outburst events, for detecting new long-period LBV binaries and the reliable determination of the orbital parameters. 

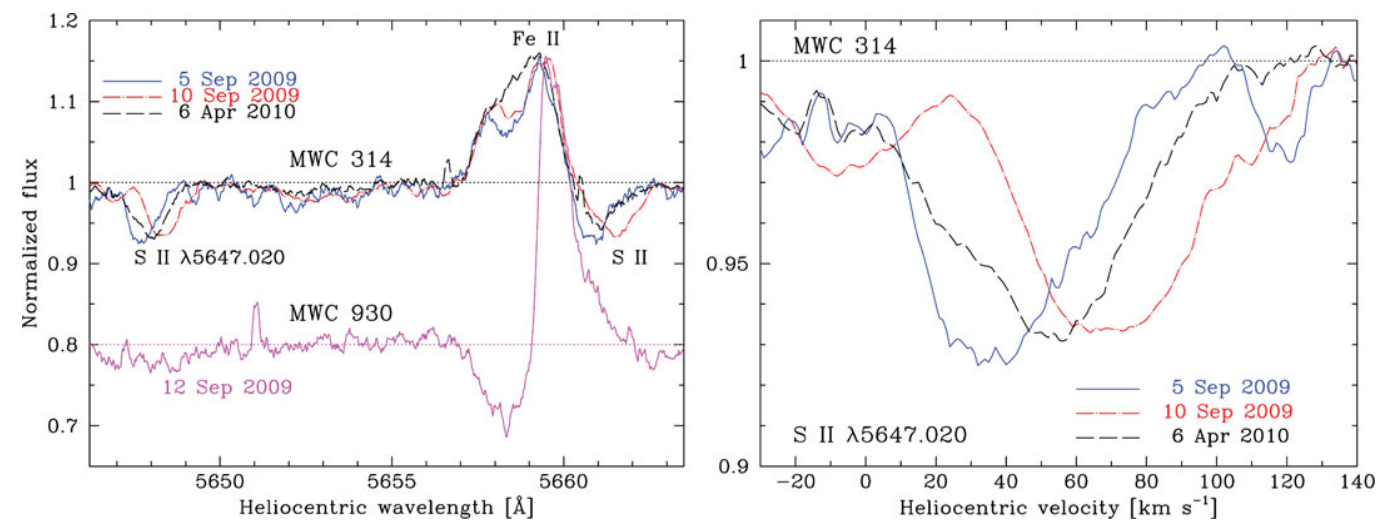

Figure 1. Large radial velocity changes of $\sim 33 \mathrm{~km} \mathrm{~s}^{-1}$ observed in S II lines of MWC 314 over 5 days with Mercator-HERMES confirm its LBV binary nature (see text).

\section{The LBV Binary MWC 314 and LBV Candidate MWC 930}

MWC 314 was observed with HERMES on 5 and 10 Sep 2009, and on 6 April 2010. Muratorio et al. (2008) found evidence for a $\sim 30$ day orbital period and proposed that its double-peaked permitted and forbidden ionic emission lines (Fe II, Cr II, Ti II) are formed in a warm disk rotating around the star. Its orbital parameters are however still unknown at present. We observe with HERMES many broad and double-peaked optical emission lines (i.e., Fe II $\lambda 5657$ ) reminiscent of a Be-star spectrum. The emission line flux maxima are strongly variable due to wind opacity changes in the emission line formation region. The radial velocities of these optical emission lines are however invariable, signaling a stable circumstellar or circumbinary (disc) envelope. We observe large changes in the radial velocity centroid of photospheric S II absorption lines from $37 \mathrm{~km} \mathrm{~s}^{-1}$ (blue curve in the right-hand panel of Fig. 1) to $70 \mathrm{~km} \mathrm{~s}^{-1}$ (red curve) in only 5 days. Our short-term spectroscopic observations with HERMES hence confirm the binarity of MWC 314 . We observe many strong optical P Cygni profiles in the LBV candidate MWC 930 in Sep 2009 (magenta curve in Fig. 1) signaling the wind properties of a massive hot star.

\section{Summary}

Long-term monitoring with Mercator-HERMES of the optical spectrum of the prototypical LBV P Cyg reveals variability at the base of its supersonic wind that can be linked to moderate $V$-changes (of $0^{\mathrm{m}} .1$ to $0^{\mathrm{m}} .2$ ) over a period of $\sim 4$ months. We find strong indications for the binary nature of MWC 314 from large radial velocity changes observed in photospheric absorption lines during less than one week. We observe prominent P Cygni profiles in the optical spectrum of LBV candidate MWC 930, signaling the presence of a central massive hot star. The optical spectral lines of LBV candidate HD 168625 are less variable, although we also observe clear signatures of expanding H $\alpha$ wind variability on short time-scales of $\sim 1$ month. The optical spectrum of HD 168607 reveals large line profile changes over the past 12 years confirming its LBV designation.

\section{References}

Raskin, G. \& Van Winckel, H. 2008, in: I. S. McLean \& M. M. Casali (eds.), Optical and Infrared Interferometry, SPIE Conference Series 7014, p. 178

Muratorio, G., Rossi, C., \& Friedjung, M. 2008, A\& $A, 487,637$ 